\title{
Local modal participation analysis of nonlinear systems using Poincaré linearization
}

\author{
Boumediene Hamzi • Eyad H. Abed
}

Received: 2 March 2019 / Accepted: 9 November 2019 / Published online: 26 November 2019

(C) The Author(s) 2019

\begin{abstract}
The paper studies an extension to nonlinear systems of a recently proposed approach to the definition of modal participation factors. A definition is given for local mode-in-state participation factors for smooth nonlinear autonomous systems. While the definition is general, the resulting measures depend on the assumed uncertainty law governing the system initial condition, as in the linear case. The work follows Hashlamoun et al. (IEEE Trans Autom Control 54(7):14391449 2009) in taking a mathematical expectation (or set-theoretic average) of a modal contribution measure over an uncertain set of system initial state. Poincaré linearization is used to replace the nonlinear system with a locally equivalent linear system. It is found that under a symmetry assumption on the distribution of the initial state, the tractable calculation and analytical formula for mode-in-state participation factors found for the linear case persists to the nonlinear setting. This paper is dedicated to the memory of Professor Ali $\mathrm{H}$. Nayfeh.
\end{abstract}

\section{B. Hamzi $(\varangle)$}

Department of Mathematics, Imperial College London, London, UK

e-mail: boumediene.hamzi@gmail.com

E. H. Abed

Department of Electrical and Computer Engineering and the Institute for Systems Research, University of Maryland, College Park, MD 20742, USA

e-mail: abed@umd.edu
Keywords Participation factors - Nonlinear systems . Poincaré linearization

\section{Introduction}

We study local modal participation analysis for nonlinear autonomous systems in the vicinity of an equilibrium point. This work builds on [1], which proposed new definitions of modal participation factors for linear systems.

In the early 1980s, Verghese et al. [2,3] introduced quantities they referred to as modal participation factors. These quantities have been used widely, especially in the electric power systems field. In 2009, the authors of [1] presented a new approach to the fundamental definition of modal participation factors, building on their previous paper [4]. The idea of modal participation factors, which will be reviewed further in the next section, is to give measures of the relative contribution of system modes in system states, and of system states in system modes. In [1], such measures are developed by taking an average of relative contribution measures over an uncertain set of system initial conditions. The idea is that fixing the system initial condition affects the modal participations, and that initial conditions are in reality uncertain, indeed possibly random due to inherent noise. Indeed, if one takes a view that the initial time also is not fixed, noise can be viewed as having the effect of allowing the initial condition to be reset over time, effectively allowing the initial 
condition to explore a neighborhood of an equilibrium point over a short-time interval. By taking an averaging approach, the authors of [1] find that a dichotomy arises in this new view of modal participation factors. In this dichotomy, participation factors measuring mode-instate participation need to be viewed as distinct from participation factors measuring state-in-mode participation. This dichotomy was not recognized prior to [1], and a single formula was previously used to quantify both types of modal participation.

In [1], it was found that analytical formulas for mode-in-state participation factors fell out of the analysis very nicely, under basic symmetry assumptions on the distribution of the initial state. However, even under the same symmetry assumptions on the initial state, state-in-mode participation factors were not easy to calculate analytically, and formulas that could be derived were more complicated than for the mode-instate case.

Here, we explore extension of the work in [1] to the nonlinear setting, focusing on behavior near an equilibrium point. We are able to give an analysis and derivation of formulas for mode-in-state participation factors (under basic symmetry assumptions as in the linear case). Rather than using Jacobian linearization and applying the work of [1] to the linearized system, we employ Poincaré linearization, which gives an exact construction of a locally equivalent linear system. This work also follows a different approach to define participation factors for nonlinear models than that pursued in [5], where modal participation for a nonlinear system was defined and studied using a fixed initial state using Taylor series methods and state trajectory estimation.

Before proceeding to the main developments of the paper, it is perhaps useful to provide a brief discussion of studies on modal participation, addressing motivation of researchers on this topic, the various approaches taken in different disciplines, and applications that have been pursued.

The present work is motivated by the original work of Verghese et al. [2,3] that was mentioned above. The authors of $[2,3]$ introduced their notion of modal participation factors as a tool to aid in modal analysis of large power grids. Oscillatory modes are common in power systems, and it is important to have systematic tools for their analysis. Since power grids consist of interconnected areas and can cover large expenses of territory (indeed entire continents), engineers are naturally interested in obtaining reduced models that cap- ture modes of special interest. The modal participation factors of [2,3] were employed for this purpose, in an overarching framework that the authors referred to as selective modal analysis (SMA). (A recent review of SMA is [6].) In addition, modal analysis in a power grid should provide tools for determining the best sites for insertion of actuators to control modes that may be troubling or dangerous, or for determining the best locations for placing measurement devices that allow system operators to monitor such modes in real time. A recent application of the concepts in [2,3] to power systems with significant levels of renewable generation is given in [7]. Early examples of work on actuator placement in power networks using the original modal participation concept include [8,9]. Recent examples of modal participation studies in power systems motivated by the more recent approach [1] include [10-13]. The approach has also been applied in power electronics [14] and electromagnetic devices [15].

The term modal participation factors is also commonly used in the field of structural analysis, with applications in mechanical, aerospace, and civil engineering. The concept of modal participation factors introduced in electric power engineering in $[2,3]$ was developed independently of the notion used in structural analysis. Modal participation factors as studied in structural analysis have been used, for example, to study vibrations of tall buildings [16]. An example of use of the basic modal participation factors concept from structural analysis in aerospace engineering is [17]. A distinction between the two themes of modal participation factors in electric power engineering and in structural analysis is the focus on the impact of forcing functions on modal response in the structural analysis literature. In contrast, in the electric power literature, a large ostensibly autonomous dynamic system is considered (representing the power grid). Bridging between these concepts could be a fruitful area for future investigation. The concepts of modal participation factors in both electrical and mechanical engineering are not absolute by any means, as they are definitions that deemed suitable for various purposes by their authors. Later, researchers sometimes propose modifications to address perceived needed improvements. For example, in structural analysis, Chopra [18] introduced a new notion of modal participation factor aiming to make a major improvement to the standard definition used in that field. Similarly, the original concept of $[2,3]$ in electric power engineering has been revisited in [1], as 
noted above. The paper [1] followed an earlier initial study by the same group, [4].

The remainder of the paper proceeds as follows. In Sect. 2, needed background material is recalled. In Sect. 3, mode-in-state participation factors are defined for nonlinear systems in the vicinity of an equilibrium point, under a symmetry assumption on the uncertainty in the system initial condition. Conclusions and issues for further research are discussed in Sect. 4.

\section{Background}

\subsection{Participation factors for linear systems: original approach [2,3]}

Let $\Sigma_{L}$ denote the linear time-invariant system

$$
\Sigma_{L}: \dot{x}=A x
$$

where $x \in \mathbb{R}^{n}$ and the state dynamics matrix $A \in \mathbb{R}^{n \times n}$ has $n$ distinct eigenvalues $\lambda_{i}, i=1, \ldots, n$.

The system state $x(t)$ of course consists of a linear combination of exponential functions

$x^{i}(t)=\mathrm{e}^{\lambda_{i} t} c^{i}$,

where the vectors $c^{i}$ are determined by the system's initial condition $x(0)$. These functions are the system modes and are useful in modal analysis of linear systems.

Let $r^{i}$ be the right eigenvector of the matrix $A$ associated with eigenvalue $\lambda_{i}, i=1, \ldots, n$, and let $\ell^{i}$ be the left(row) eigenvector of $A$ associated with the eigenvalue $\lambda_{i}, i=1, \ldots, n$. The right and left eigenvectors are taken to satisfy the normalization

$\ell^{i} r^{j}=\delta_{i j}$,

where $\delta_{i j}=1$ if $i=j$ and $\delta_{i j}=0$ if $i \neq j$. We note that while the normalization (3) is common in bifurcation analysis, it does not completely specify the eigenvectors. The normalization is easily augmented to uniquely determine the left and right eigenvectors, say for instance by requiring the first nonzero element of the right eigenvectors to be 1 .

Given a linear system $\dot{x}=A x$ with initial condition $x(0)=x^{0}$, its solution can be written as

$$
x(t)=\mathrm{e}^{A t} x^{0}=\sum_{i=1}^{n}\left(\ell^{i} x^{0}\right) \mathrm{e}^{\lambda_{i} t} r^{i} .
$$

The $k$ th state variable evolves according to

$x_{k}(t)=\left(\mathrm{e}^{A t} x^{0}\right)_{k}=\sum_{i=1}^{n}\left(\ell^{i} x^{0}\right) \mathrm{e}^{\lambda_{i} t} r_{k}^{i}$

Using these facts and taking specific initial conditions, Verghese et al. [2,3] motivated the following definition of the quantities $p_{k i}$ which they named modal participation factors:

$p_{k i}:=\ell_{k}^{i} r_{k}^{i}$

Choosing the initial condition to be $x^{0}=e_{k}$, the unit vector on the $k$ th coordinate axis, the quantities $p_{k i}$ gained the interpretation of mode-in-state participation factors. The scalars $p_{k i}$ are dimensionless. Moreover, using a coordinate transformation to focus on the system modes and taking the initial condition to be $x^{0}=r^{k}$, the right eigenvector corresponding to $\lambda_{i}$, the quantities $p_{k i}$ were also given an interpretation as mode-in-state participation factors. This is the reason that it is very common in papers and books using modal participation factors to interchangeably refer to participation of modes in states and participation of states in modes, always using the same formula (6).

\subsection{Participation factors for linear systems: more recent approach ([1])}

In [1], simple examples were used to motivate the need for new definitions (and associated derivations of formulas) for modal participation factors. The examples illustrated that there is a weakness in the original definitions, as they give the same quantitative values for mode-in-state participation factors and state-in-mode participation factors, and these values were at odds with basic intuition in some of the examples. The examples showed that, especially when calculating the contribution of system states in system modes, formula (6) may fall short of giving an intuitively acceptable result. Thus, new fundamental definitions were given based on averaging over the system initial condition.

The linear system

$\dot{x}=A x$

usually represents the small perturbation dynamics near an equilibrium. The initial condition for such a perturbation is usually viewed as being an uncertain vector of small norm. In [1], new definitions of mode-instate and state-in-mode participation factors were given 
using deterministic and probabilistic uncertainty models for the initial condition. These definitions employed averaging (deterministic or stochastic, as appropriate depending on the initial condition uncertainty model) over the uncertain initial condition. In the sequel, we proceed along the lines set in [1], focusing on the case of mode-in-state participation factors for nonlinear systems. Very recently, Iskakov $[19,20]$ has proposed a modification of the approach in [1], retaining the basic averaging approach but incorporating modal and state energies into the modified definition. The focus in $[19,20]$ is on state-in-mode participation factors, where the modified energy-based definition is shown to remove a sensitivity that occurs for the complex eigenvalues case to the specific eigenvector pairs selected under the normalization (3). This appears to result in improved results especially for the case of complex eigenvalues and state-in-mode participation. Further work along the direction of employing energy measures as proposed by Iskakov is a topic for future research.

Definition 1 In the set-theoretic formulation, the participation factor measuring relative influence of the mode associated with $\lambda_{i}$ on state component $x_{k}$ is

$p_{k i}:=\underset{x^{0} \in \mathcal{S}}{\operatorname{avg}} \frac{\left(\ell^{i} x^{0}\right) r_{k}^{i}}{x_{k}^{0}}$

whenever this quantity exists. Here, $x_{k}^{0}=\sum_{i=1}^{n}\left(\ell^{i} x^{0}\right) r_{k}^{i}$ is the value of $x_{k}(t)$ at $t=0$, and " $\operatorname{avg}_{x^{0} \in \mathcal{S}}$ " is an operator that computes the average of a function over a set $\mathcal{S} \subset R^{n}$ (representing the set of possible values of the initial condition $x^{0}$ ).

With a probabilistic description of the uncertainty in the initial condition $x^{0}$, the average in (8) is replaced by a mathematical expectation:

Definition 2 The general formula for the participation factor $p_{k i}$ measuring participation of mode $i$ in state $x_{k}$ becomes

$p_{k i}:=E\left\{\frac{\left(\ell^{i} x^{0}\right) r_{k}^{i}}{x_{k}^{0}}\right\}$

where the expectation is evaluated using some assumed joint probability density function $f\left(x^{0}\right)$ for the initial condition uncertainty. (Of course, this definition applies only when the expectation exists.)
In [1], it was found that both definition 8 and definition 9 lead to a simple result that agrees with Eq. (6) under a symmetry assumption on the uncertainty in the initial condition. In the set-theoretic definition, the symmetry assumption is that the initial condition uncertainty set $\mathcal{S}$ is symmetric with respect to each of the hyperplanes $\left\{x_{k}=0\right\}, k=1, \ldots, n$. In the probabilistic setting of Definition 2, the assumption is that the initial condition components $x_{1}^{0}, x_{2}^{0}, \ldots, x_{n}^{0}$ are independent random variables with marginal density functions which are symmetric with respect to $x_{k}^{0}=0$, $k=1,2, \ldots, n$, or are jointly uniformly distributed over a sphere centered at the origin. Under either the set-theoretic or probabilistic symmetry assumption, it was found in [1] that the same expression originally introduced by Verghese et al. [2,3] results as a measure of mode-in-state participation factors:

$p_{k i}=\ell_{k}^{i} r_{k}^{i}$.

\subsection{State-in-mode participation factors}

Hashlamoun, Hassouneh and Abed [1] also gave similar set-theoretic and probabilistic definitions for modein-state participation factors for linear systems. The calculations were found to be less straightforward than for the mode-in-state participation factors setting, even under a symmetry assumption on the initial condition. We will not recall the details of the development of state-in-mode participation factors for linear systems from [1]. It will suffice to note the situation for the case of distinct real eigenvalues to have an idea of the results.

Definition 3 The participation factor of state $x_{k}$ in mode $i$ is

$\pi_{k i}:=\mathrm{E}\left\{\frac{\ell_{k}^{i} x_{k}^{0}}{\sum_{j=1}^{n}\left(\ell_{j}^{i} x_{j}^{0}\right)}\right\}=\mathrm{E}\left\{\frac{\ell_{k}^{i} x_{k}^{0}}{z_{i}^{0}}\right\}$,

whenever this expectation exists, where $z_{i}^{0}=z_{i}(0)=$ $\ell^{i} x^{0}$, and where $z_{i}(t)$ is the $i$ th system mode

$z_{i}(t)=\mathrm{e}^{\lambda_{i} t} \ell^{i} x^{0}=\mathrm{e}^{\lambda_{i} t} \sum_{j=1}^{n}\left(\ell_{j}^{i} x_{j}^{0}\right)$.

It is shown in [1] that

$$
\begin{aligned}
\pi_{k i} & =\mathrm{E}\left\{\frac{\ell_{k}^{i} x_{k}^{0}}{\sum_{j=1}^{n}\left(\ell_{j}^{i} x_{j}^{0}\right)}\right\} \\
& =\ell_{k}^{i} r_{k}^{i}+\sum_{j=1, j \neq i}^{n} \ell_{k}^{i} r_{k}^{j} E\left\{\frac{z_{j}^{0}}{z_{i}^{0}}\right\}
\end{aligned}
$$


Note that the first term in the expression for $\pi_{k i}$ coincides with $p_{k i}$, the original participation factors formula. However, the second term does not vanish in general. This is true even when the components $x_{1}^{0}, x_{2}^{0}, \ldots, x_{n}^{0}$ representing the initial conditions of the state are assumed to be independent. Assuming that the units of the state variables have been scaled to ensure that the probability density function $f\left(x^{0}\right)$ is such that the components $x_{1}^{0}, x_{2}^{0}, \ldots, x_{n}^{0}$ are jointly uniformly distributed over the unit sphere in $R^{n}$ centered at the origin, modal participation factors were evaluated in [1] using Definition 3, yielding the following explicit formula that is applicable under the foregoing uncertainty model for the system initial state.

Proposition 1 ([1]) Under the assumption that the initial condition has a uniform probability density on a sphere centered at the origin, the participation factor of state $x_{k}$ in mode $i$ is

$\pi_{k i}=\ell_{k}^{i} r_{k}^{i}+\sum_{j=1, j \neq i}^{n} \ell_{k}^{i} r_{k}^{j} \frac{l^{j}\left(\ell^{i}\right)^{T}}{\ell^{i}\left(\ell^{i}\right)^{T}}$.

\subsection{Poincaré linearization}

Poincaré linearization is a well-known technique for transforming an autonomous nonlinear system into a locally equivalent linear system via diffeomorphism. The technique is useful in this paper for extending the definitions of mode-in-state participation factors proposed in [1] to the nonlinear setting. In the following, we review the technique.

Consider a nonlinear ODE

$\dot{x}=f(x)$,

where $x \in \mathbb{R}^{n}$ and $f$ is an analytic vector field on $\mathbb{R}^{n}$. Let $A=\left.\frac{\partial f}{\partial x}\right|_{x=0}$ be the Jacobian of $f$ at the origin.

Definition 4 ([21]) Given a matrix $A \in \mathbb{R}^{n \times n}$ with eigenvalues $\lambda_{i}, i=1, \ldots, n$, we say that the $n$-tuple $\lambda=\left(\lambda_{1}, \ldots, \lambda_{n}\right)$ is resonant if among the eigenvalues there exists a relation of the form

$(m, \lambda)=\sum_{k=1}^{n} m_{k} \lambda_{k}=\lambda_{s}$,

where $m=\left(m_{1}, \ldots, m_{n}\right), m_{k} \geq 0, \sum_{k} m_{k} \geq 2$. Such a relation is called a resonance. The number $|m|=$ $\sum_{k=1}^{n} m_{k}$ is called the order of the resonance.
Example 1 ([21]) The relation $\lambda_{1}=2 \lambda_{2}$ is a resonance of order 2 ; the relation $2 \lambda_{1}=3 \lambda_{2}$ is not a resonance; the relation $\lambda_{1}+\lambda_{2}=0$, or equivalently $\lambda_{1}=2 \lambda_{1}+\lambda_{2}$, is a resonance of order 3 .

Theorem 1 (Poincaré's Theorem) ([21]) If the eigenvalues of the matrix A are nonresonant, then the nonlinear $O D E$

$\dot{x}=A x+O\left(\|x\|^{2}\right)$

can be reduced to the linear $O D E$

$\dot{y}=A y$

by a formal change of variable $x=y+\cdots$. (The dots denote series starting with terms of degree two or higher.)

If the $n$-tuple $\lambda=\left(\lambda_{1}, \ldots, \lambda_{n}\right)$ is resonant, we will say that

$x^{m}:=x_{1}^{m_{1}} \cdots x_{n}^{m_{n}} e_{s}$

is resonant if $\lambda_{s}=(m, \lambda),|m| \geq 2$ with $e_{i}$ a vector in the eigenbasis of $A$ and $x_{i}$ are the coordinates with respect to the basis $e_{i}$. For example, for the resonance $\lambda_{1}=2 \lambda_{2}$, the unique resonant monomial is $x_{2}^{2} e_{1}$. For the resonance $\lambda_{1}+\lambda_{2}=0$, all monomials $\left(x_{1} x_{2}\right)^{k} x_{s} e_{s}$ are resonant [21].

Theorem 2 (Poincaré-Dulac Theorem [21])) If the eigenvalues of the matrix A are resonant, then the nonlinear $O D E$

$\dot{x}=A x+\cdots$

can be reduced to the $O D E$

$\dot{y}=A y+w(y)$

by a formal change of variable $x=y+\cdots$ (the dots denote series starting with terms of degree two or higher), where all monomials in the series $w$ are resonant.

There are also known convergence results associated with Poincare linearization, of which the following is the most well-known.

Theorem 3 (Poincaré-Siegel) Suppose the eigenvalues $\left\{\lambda_{i}\right\}, i=1, \ldots, n$, of the linear part of an analytic vector field at an equilibrium point are nonresonant and either $\operatorname{Re}\left(\lambda_{i}\right)>0, i=1, \ldots, n$ or $\operatorname{Re}\left(\lambda_{i}\right)<0$, $i=, \ldots, n$, or the $\left(\lambda_{i}\right)$ satisfy the Siegel condition, i.e., 
are such that there exists $C>0$ and $v$ such that for all $i=1, \ldots, n$

$\left|\lambda_{i}-(m, \lambda)\right| \geq \frac{C}{|m|^{v}}$

for all $m=\left(m_{1}, \ldots, m_{n}\right)$, where $\left(m_{i}\right)$ are nonnegative integers with $|m|=\sum_{i=1}^{n} m_{i} \geq 2$. Then, the power series in Poincaré's theorem converges on some neighborhood of the equilibrium point.

Remark 1 There are also some convergence results in the case of resonant eigenvalues; the reader is encouraged to consult [21] for further details on Poincaré linearization.

\subsection{Hartman-Grobman theorem}

Another very important result in the local qualitative theory of nonlinear ordinary differential equations is the Hartman-Grobman theorem, which says that near a hyperbolic equilibrium point $x^{e}$, the nonlinear system (14) has the same qualitative structure as the linear system (17).

Theorem 4 [22] Let $E$ be an open subset of $\mathbb{R}^{n}$ containing the origin, let $f \in C^{1}(E)$, and let $\phi_{t}$ be the flow of the nonlinear system (14). Suppose that $f(0)=0$ and that the matrix $A=D f(0)$ has no eigenvalue with zero real part. Then, there exists a homeomorphism $\varphi$ of an open set $U$ containing the origin onto an open set $V$ containing the origin such that for each $x^{0} \in U$, there is an open interval $I_{0} \subset \mathbb{R}$ containing zero such that for all $x^{0} \in U$ and $t \in I_{0}$

$\varphi \circ \phi_{t}\left(x^{0}\right)=\mathrm{e}^{A t} \varphi\left(x^{0}\right)$,

i.e., $\varphi$ maps trajectories of (14) near the origin onto trajectories of (17) near the origin.

\section{Mode-in-state participation factors for nonlinear systems}

Consider a nonlinear ODE

$\dot{x}=f(x)$

with $f \in \mathcal{C}\left(\mathbb{R}^{n} ; \mathbb{R}^{n}\right), f(0)=0$, and consider the Taylor expansion of $f$ around the origin

$\dot{x}=A x+\tilde{f}^{[2]}(x)+O\left(\|x\|^{3}\right)$

where $A=\left.\frac{\partial f}{\partial x}\right|_{x=0}$ and $\tilde{f}^{[2]}$ represents terms of order 2 in the expansion of $f(x)$. We have the following result.
Theorem 5 If the eigenvalues of $A$ are nonresonant (resp. satisfy one of the conditions of the PoincaréSiegel theorem), then there exists a diffeomorphism that formally (resp. analytically) transforms the nonlinear ODE (22) into a linear ODE. In this case, the mode-instate participation factors of (22) are the same as those of the linearized system $\dot{x}=A x$.

Proof First, we normalize $A$ using the change of coordinates

$z=V^{-1} x$,

where $V=\left[r^{1} r^{2} \cdots r^{n}\right]$ represents the matrix of right eigenvectors of $A$. Under the change of coordinates (24), the ODE (23) becomes

$$
\begin{aligned}
\dot{z} & =\Lambda z+V^{-1} \tilde{f}^{[2]}\left(V^{-1} z\right)+O\left(\|z\|^{3}\right) \\
& :=\Lambda z+f^{[2]}(z)+O\left(\|z\|^{3}\right)
\end{aligned}
$$

Next, we normalize the higher-order terms through the change of coordinates

$$
\begin{aligned}
\tilde{z} & =\phi(z)=z+\phi^{[2]}(z)+O\left(\|z\|^{3}\right) \\
& =z+z^{T}\left[\begin{array}{c}
P_{1} \\
\vdots \\
P_{n}
\end{array}\right] z+O\left(\|z\|^{3}\right)
\end{aligned}
$$

where $\phi \in \mathcal{C}\left(\mathbb{R}^{n} ; \mathbb{R}^{n}\right)$. Using Poincaré linearization, we know that if the eigenvalues of $A$ are nonresonant, then there is a formal change of coordinates $\phi$ such that the trajectories of (22) are locally diffeomorphic to the trajectories of

$\dot{\tilde{z}}=\Lambda \tilde{z}$

$$
\text { If } \Lambda=\left.\operatorname{diag}\left(\lambda_{i}\right)\right|_{i=1} ^{n} \text {, then }
$$

$\tilde{z}(t)=\mathrm{e}^{\Lambda t} \tilde{z}(0)$,

whose $i$ th component is

$\tilde{z}_{i}(t)=\mathrm{e}^{\lambda_{i} t} \tilde{z}_{i}(0)$.

Using (26), we get $z(t)=\phi^{-1}\left(\mathrm{e}^{\Lambda t} \phi\left(z^{0}\right)\right)$, which can be rewritten as

$$
\begin{gathered}
z(t)=\mathrm{e}^{\Lambda t} \phi\left(z^{0}\right)-\phi(z(0))^{T} \mathrm{e}^{\Lambda^{t} t}\left[\begin{array}{c}
P_{1} \\
\vdots \\
P_{n}
\end{array}\right] \\
\mathrm{e}^{\Lambda t} \phi(z(0))+O\left(\|z\|^{3}\right),
\end{gathered}
$$


and

$z_{i}(t)=\mathrm{e}^{\lambda_{i} t} \phi_{i}\left(z^{0}\right)-\phi^{T}\left(z^{0}\right) \mathrm{e}^{\Lambda^{T} t} P_{i} \mathrm{e}^{\Lambda t} \phi\left(z^{0}\right)+\cdots$

Using (24), we get

$$
\begin{aligned}
x_{k}(t)= & {\left[r^{1} \cdots r^{n}\right]_{k \text { th row }}\left[\begin{array}{c}
z_{1} \\
\vdots \\
z_{n}
\end{array}\right]=\sum_{i} r_{k}^{i} z_{i}(t) } \\
= & \sum_{i} r_{k}^{i}\left(\mathrm{e}^{\lambda_{i} t} \phi_{i}\left(z^{0}\right)-\phi^{T}\left(z^{0}\right) P_{i} \phi\left(z^{0}\right)\right) \\
& +\cdots
\end{aligned}
$$

It is instructive to consider the linear case first. We set $P_{i}=0$, and the higher-order terms are also set to zero in (26). This gives

$x_{k}(t)=\sum_{i=1}^{n} r_{k}^{i} \mathrm{e}^{\lambda_{i} t} \phi_{i}\left(z^{0}\right)=\sum_{i=1}^{n} r_{k}^{i} \mathrm{e}^{\lambda_{i} t} \ell^{i} x^{0}$

Then, the participation of the $\mathrm{e}^{\lambda_{i} t}$ mode-in-state $x_{k}(t)$ is

$p_{k i}:=\left.\operatorname{avg} \frac{\mathrm{e}^{\lambda_{i} t} r_{k}^{i} \ell^{i} x^{0}}{x_{k}(t)}\right|_{t=0}=\ell_{k}^{i} r_{k}^{i}$

(agreeing, of course, with the previous calculation of [1] in the linear case [1]).

Next, we consider the nonlinear setting, where we assume that $P_{i} \neq 0$. The participation of $\mathrm{e}^{\lambda_{i} t}$ in $x_{k}(t)$ is obtained using the set-theoretic definition as follows (quantities are evaluated at time $t=0$ ): $\left.\operatorname{avg} \frac{\mathrm{e}^{\lambda_{i} t} r_{k}^{i} \phi_{i}\left(z^{0}\right)}{x_{k}(t)}\right|_{t=0}=\operatorname{avg}$

$\left.\frac{\mathrm{e}^{\lambda_{i} t} r_{k}^{i} \phi_{i}\left(z^{0}\right)}{\sum_{i=1}^{n} r_{k}^{i}\left(\mathrm{e}^{\lambda_{i} t} \phi_{i}\left(z^{0}\right)-\sum_{j, m} \theta_{j, m} \mathrm{e}^{\left(\lambda_{j}+\lambda_{m}\right) t}\right)}\right|_{t=0}$

Since $\phi_{i}\left(z^{0}\right)=\ell^{i} x^{0}+\cdots$, then

$$
\begin{aligned}
\sum_{j, m} \theta_{j, m} & =\sum_{j, m} \phi_{j}\left(z^{0}\right) \phi_{m}\left(z^{0}\right) p_{j, m} \\
& =\sum_{j, m}\left(\ell^{j} x^{0}\right)\left(\ell^{m} x^{0}\right) p_{j, m}
\end{aligned}
$$

Hence, the participation of the mode $\mathrm{e}^{\lambda_{i} t}$ in $x_{k}(t)$ is

$$
\begin{aligned}
p_{k i} & :=\left.\operatorname{avg} \frac{\mathrm{e}^{\lambda_{i} t} r_{k}^{i} \phi_{i}\left(z^{0}\right)}{x_{k}(t)}\right|_{t=0} \\
& =\left.\operatorname{avg} \frac{\mathrm{e}^{\lambda_{i} t} r_{k}^{i} \phi_{i}\left(z^{0}\right)}{\sum_{i=1}^{n} r_{k}^{i}\left(\mathrm{e}^{\lambda_{i} t} \phi_{i}\left(z^{0}\right)-\sum_{j, m} \theta_{j, m} \mathrm{e}^{\left(\lambda_{j}+\lambda_{m}\right) t}\right)}\right|_{t=0} \\
& =r_{k}^{i} \ell_{k}^{i} .
\end{aligned}
$$

Under the assumptions made, the mode-in-state participation factors are seen to agree with those of the linearized system.

Examples Consider a nonlinear system whose linear part is from an example in [1]:

$\dot{x}=\underbrace{\left[\begin{array}{ll}a & b \\ 0 & d\end{array}\right]}_{A_{1}} x+\Psi(x)$,

with $\Psi$ a polynomial of order $N \geq 2$. If $a \neq m \cdot d$ for any $m \in \mathbb{N}$, then the eigenvalues of the matrix $A_{1}$ are nonresonant and, therefore, by the Poincaré's theorem, there exists a formal transformation that transforms (37) to

$\dot{z}=A z$.

Furthermore, if $\lambda_{1}=a$ and $\lambda_{2}=d$ satisfy one of the conditions of the Poincaré-Siegel theorem, then the transformation is analytic. In both cases, the mode-instate participation factors of (37) are locally equal to the mode-in-state participation factors of the linear system (38).

A similar result holds for the following nonlinear system, whose linear part is from another example of [1]:

$\dot{x}=\underbrace{\left[\begin{array}{cc}1 & 1 \\ -d-d\end{array}\right]}_{A_{1}} x+\Psi(x)$,

with $d \neq 1$ (nonresonance condition) and $\Psi$ is a polynomial of order $N \geq 2$.

If the eigenvalues are resonant, and the origin is hyperbolic, we can still say something on the modein-state participation factors.

Theorem 6 If the origin is a hyperbolic point, then there exists a homeomorphism that transforms the nonlinear ODE (22) into the linear ODE (27) [22]. In this case, the mode-in-state participation factors of (22) are the same as those of the linearized system $\dot{x}=A x$.

Proof First, we normalize $A$ using the change of coordinates (24) where $V=\left[r^{1} r^{2} \ldots r^{n}\right]$ represents the matrix of right eigenvectors of $A$. Under the homeomorophism in the Hartman-Grobman theorem, the ODE (22) becomes

$\dot{z}=\Lambda z$

The proof regarding mode-in-state participation factors comes directly from applying the result for the linear case in Sect. 3. 
Example [22] Consider the system

$\dot{y}=-y$

$\dot{z}=z+y^{2}$

It is shown in [22] that with the homeomorphism

$\phi(y, z)=\left[\begin{array}{c}y \\ z+\frac{y^{2}}{3}\end{array}\right]$

the solution of (41)-(42) is homeomorphic to the solution of

$\dot{y}=-y$

$\dot{z}=z$

and, therefore, the mode-in-state participation factors of the nonlinear system are the same as those of the linearized system.

\section{Conclusion}

There is a dichotomy in modal participation for linear systems. Hence, we expect a similar dichotomy for nonlinear systems. Participation of modes in states is relatively easy to evaluate using averaging over an uncertain set of initial conditions assuming symmetric uncertainty. The mode-in-state participation formulas under these circumstances were found to be the same for a nonlinear system as for its linearization, assuming the nonresonance condition. While this may be viewed as not necessarily surprising, it was necessary to perform the calculation and report the obtained conclusions. What might transpire under different assumptions on the initial condition uncertainty has not been addressed in the paper, and could be investigated in the future. Moreover, participation of states in modes for nonlinear systems may also agree with the analogous measures for the linearized system, but without explicit calculation, this is not clear. In addition, extending to nonlinear systems the definitions and formulas for state-in-mode participation factors given in [1] using a recently proposed modal energy approach $[19,20]$ is a topic worthy of further study. Besides calculation of state-in-mode participation factors, some other issues that could be considered in future work are: computing modal participation factors for nonlinear systems from data and using the Frobenius-Perron operator to compute these measures. Another extension is to consider any possible relationship of nonlinear eigenvalues and eigenvectors for nonlinear systems
$[23,24]$ to modal participation analysis for nonlinear systems. A preliminary version of this work appeared in [25].

Acknowledgements $\mathrm{BH}$ thanks the European Commission and the Scientific and the Technological Research Council of Turkey (Tubitak) for financial support received through Marie Curie Fellowships. EHA thanks the US Air Force Office of Scientific Research for partial support under Grant \#FA9550-09-10538 .

\section{Compliance with ethical standards}

Conflict of interest The authors declare that they have no conflict of interest.

Open Access This article is distributed under the terms of the Creative Commons Attribution 4.0 International License (http:// creativecommons.org/licenses/by/4.0/), which permits unrestricted use, distribution, and reproduction in any medium, provided you give appropriate credit to the original author(s) and the source, provide a link to the Creative Commons license, and indicate if changes were made.

\section{References}

1. Hashlamoun, W.A., Hassouneh, M.A., Abed, E.H.: New results on modal participation factors: revealing a previously unknown dichotomy. IEEE Trans. Autom. Control 54(7), 1439-1449 (2009)

2. Pérez-Arriaga, I.J., Verghese, G.C., Schweppe, F.C.: Selective modal analysis with applications to electric power systems, Part I: heuristic introduction. IEEE Trans. Power Appar. Syst. 101(9), 3117-3125 (1982)

3. Verghese, G.C., Pérez-Arriaga, I.J., Schweppe, F.C.: Selective modal analysis with applications to electric power systems, Part II: the dynamic stability problem. IEEE Trans. Power Appar. Syst. 101(9), 3126-3134 (1982)

4. Abed, E.H., Lindsay, D., Hashlamoun, W.A.: On participation factors for linear systems. Automatica 36(10), 14891496 (1999)

5. Jang, G., Vittal, V., Kliemann, W.: Effect of nonlinear modal interaction on control performance: use of normal forms technique in control design. I. General theory and procedure. IEEE Trans. Power Syst. 13(2), 401-407 (1998)

6. Rouco, L., Pagola, F.L., Verghese, G.C., Pérez-Arriaga, I.J.: Selective modal analysis. In: Chow, J.H. (ed.) Power System Coherency and Model Reduction. Springer, New York (2013)

7. Setiadia, H., Krismantoa, A.U., Mithulananthana, N., Hossain, M.J.: Modal interaction of power systems with high penetration of renewable energy and BES systems. Int. J. Electr. Power Energy Syst. 97, 385-395 (2018)

8. Gao, B., Morison, G.K., Kundur, P.: Voltage stability evaluation using modal analysis. IEEE Trans. Power Syst. 7(4), 1529-1542 (1992)

9. Mansour, Y., Xu, W., Alvarado, F., Rinzin, C.: SVC Placement using critical modes of voltage stability. IEEE Trans. Power Syst. 9(2), 757-763 (1994) 
10. Ramos, R.A., Moraco, A.G.M., Fernandes, T.C.C., de Oliveira, R.V.: Application of extended participation factors to detect voltage fluctuations in distributed generation systems. In: Proceedings of IEEE Power and Energy Society General Meeting, pp. 1-6 (2011)

11. Moraco, A.G.M., Fernandes, T.C.C., Garcia, G.S., Ramos, R.A.: Statistical analysis for the detection of voltage fluctuations in distributed synchronous generation using extended participations factors. In: Proceedings of IEEE International Conference on Industrial Technology, pp. 457-462 (2012)

12. Song, Y., Hill, D.J., Liu, T.: State-in-mode analysis of the power flow Jacobian for static voltage stability. Int. J. Electr. Power Energy Syst. 105, 671-678 (2019)

13. Netto, M., Susuki, Y., Mili, L.: Data-driven participation factors for nonlinear systems based on Koopman mode decomposition. IEEE Control Syst. Soc. Lett. 3(1), 198-203 (2019)

14. Teerakawanich, N., Evans, P., Johnson, C.M.: Oscillation analysis of an active gate control circuit for series connected IGBTs. In: 15th International Power Electronics and Motion Control Conference, paper. DS1a.5. EPE-PEMC 2012 ECCE Europe, Novi Sad, Serbia (2012)

15. Cenedese, A., Fagherazzi, M., Bettini, P.: A novel application of selective modal analysis to large-scale electromagnetic devices. IEEE Trans. Magn. 52(3), paper no. 7203304 (2016)

16. Park, H.S., Kwon, J.H.: Optimal drift design model for multi-story buildings subjected to dynamic lateral forces. Struct. Des. Tall Spec. Build. 12, 317-333 (2003)

17. Lopez, M.J.S., Prasad, J.V.R.: Estimation of modal participation factors of linear time periodic systems using linear time invariant approximations. J. Am. Helicopter Soc. 61, paper no. 045001 (2016)
18. Chopra, A.K.: Modal analysis of linear dynamic systems: physical interpretation. J. Struct. Eng. 122, 517-527 (1996)

19. Iskakov, A.B., Yadykin, I.B.: Lyapunov modal analysis and participation factors with applications to small-signal stability of power systems. arXiv:1909.02227v1 [math.OC] (2019)

20. Iskakov, A.B.: Definition of state-In-mode participation factors for modal analysis of linear systems. Preprint (2019)

21. Arnold, V.I.: Geometrical Methods in the Theory of Ordinary Differential Equations. Springer, New York (1983)

22. Perko, L.: Differential Equations and Dynamical Systems. Springer, New York (2006)

23. Kawanoa, Y., Ohtsuka, T.: PBH tests for nonlinear systems. Automatica 80, 135-142 (2017)

24. Padoan, A., Astolfi, A.: "Eigenvalues" and "poles" of a nonlinear system: a geometric approach. In: Proceedings of the 56th IEEE Conference on Decision and Control, pp. 25752580 (2017)

25. Hamzi, B., Abed, E.: Local mode-in-state participation factors for nonlinear systems. In: Proceedings of 53rd IEEE Conference on Decision and Control (CDC), pp. 43-48 (2014)

Publisher's Note Springer Nature remains neutral with regard to jurisdictional claims in published maps and institutional affiliations. 